\title{
Hsa_circ_0004674 promotes osteosarcoma doxorubicin resistance by regulating the miR-342-3p/FBN1 axis
}

Yumei Bai ${ }^{\dagger}$, Yanghua $\mathrm{Li}^{\dagger}$, Juan Bai and Yumei Zhang*

\begin{abstract}
Background: The occurrence of chemoresistance is a common problem in tumor treatment. Circular RNA (circRNA) has been confirmed to be related to tumor chemoresistance. However, the role and the underlying molecular mechanism of hsa_circ_0004674 in the chemoresistance of osteosarcoma (OS) are still unclear.

Methods: The expression of hsa_circ_0004674, miR-342-3p, and fibrillin-1 (FBN1) was determined by qRT-PCR. Cell counting kit 8 assay was used to evaluate the doxorubicin (DXR) resistance of cells. The proliferation and apoptosis of cells were measured using colony formation assay and flow cytometry. Western blot analysis was utilized to examine the protein levels of resistance markers, $\mathrm{Wnt} / \beta$-catenin pathway markers and FBN1. The interaction between miR342-3p and hsa_circ_0004674 or FBN1 was confirmed by dual-luciferase reporter assay and RNA pull-down assay. Moreover, animal experiments were performed to assess the effect of hsa_circ_0004674 silencing on the DXR sensitive of OS in vivo.

Results: The upregulated hsa_circ_0004674 was found in DXR-resistant OS tissues and cells. Knockdown of hsa_ circ_0004674 could inhibit the DXR resistance of OS cells in vitro and promote the DXR sensitive of OS tumors in vivo. In addition, we discovered that hsa_circ_0004674 could sponge miR-342-3p, and miR-342-3p could target FBN1. MiR-342-3p inhibitor could reverse the inhibition effect of hsa_circ_0004674 knockdown on the DXR resistance of OS cells. Similarly, the suppressive effect of miR-342-3p on the DXR resistance of OS cells also could be reversed by FBN1 overexpression. Furthermore, we revealed that hsa_circ_0004674 silencing inhibited the activity of Wnt/ $\beta$-catenin pathway by the miR-342-3p/FBN1 axis.
\end{abstract}

Conclusion: Hsa_circ_0004674 facilitated the DXR resistance of OS through Wnt/ $\beta$-catenin pathway via regulating the miR-342-3p/FBN1 axis, suggesting that hsa_circ_0004674 was a promising target for the chemoresistance of OS.

Keywords: Osteosarcoma, chemoresistance, hsa_circ_0004674, miR-342-3p, FBN1

\section{Introduction}

Osteosarcoma (OS) is a malignant connective tissue tumor, and its incidence ranks first among malignant bone tumors $[1,2]$. The current treatment of OS is limb

\footnotetext{
*Correspondence: Zym707600@126.com

${ }^{\dagger}$ YumeiBai and Yanghua Li contribute to this work equally as co-first authors.

Department of Pharmacy, Jingmen No.1 People's Hospital, No.3,

Shenzhen Avenue, Duodao District, Jingmen 448000, Hubei, China
}

salvage under the protection of neoadjuvant chemotherapy, so the sensitivity of chemotherapy has an important impact on the prognosis of OS patients $[3,4]$. Drug resistance is a common cause of clinical chemotherapy failure, and its production is a complex biological process, often accompanied by many molecular interactions $[5,6]$. Therefore, elucidating the molecular mechanisms affecting chemoresistance is of great significance to the treatment and diagnosis of OS. 
Circular RNA (circRNA) contains microRNA (miRNA) binding sites, and can serve as a ceRNA to perform biological functions [7, 8]. Abnormal expression of circRNA can lead to tumorigenesis and changes in cell biological functions $[9,10]$. In addition, circRNA has been confirmed to be associated with the chemoresistance of tumors. For example, circ_0026359 could serve as miR1200 sponge to promote gastric cancer cisplatin resistance [11]. CircHIPK3 was upregulated in glioma, which could enhance the temozolomide resistance of glioma by miR-524-5p/KIF2A axis [12]. Moreover, circKDM4C was discovered to be downregulated in breast cancer, which could reduce the doxorubicin (DXR) resistance of breast cancer [13]. Therefore, circRNA is expected to be a potential target for overcoming the chemoresistance of tumors.

In previous studies, Kun-Peng et al. screened out 80 circRNAs that were differentially expressed in chemoresistance and chemosensitive OS cells by using nextgeneration sequencing analysis [14]. Among them, the significantly high expression of hsa_circ_0004674 has attracted our attention. Nevertheless, the role and molecular mechanism of hsa_circ_0004674 in OS chemoresistance are still unclear. The purpose of our study is to reveal the influence of hsa_circ_0004674 on OS DXR resistance and reveal its underlying molecular mechanism.

\section{Materials and methods Samples}

41 primary OS patients were recruited from the Jingmen No.1 People's Hospital according to the inclusion and exclusion criteria. Inclusion criteria: 1 , newly treated patients without previous surgery or chemotherapy; 2 , all patients were diagnosed with OS by histopathology; 3 , all patients and their family members were informed of this study and signed the consent form. Exclusion criteria: 1, complicated with other tumors; 2 , diseases of the nervous system; 3 , suffer from serious organ diseases. The correlation between has_circ_0004674 expression and clinicopathological characteristics of OS patients was shown in Table 1. Forty-one tumor tissues from patients with primary OS were obtained, including 18 DXR-resistant OS tissues and 23 DXR-sensitive OS tissues. This study obtained the informed consent from each patient and was approved by Jingmen No.1 People's Hospital.

\section{Cell culture and transfection}

Human OS cell lines (U2OS and KHOS) were obtained from Biovector (Beijing China) and were cultured in DMEM (Gibco, Gran Island, NY, USA) containing 10\% FBS (Gibco) and 1\% penicillin-streptomycin (Invitrogen, Carlsbad, CA, USA) at $37^{\circ} \mathrm{C}$ with $5 \% \mathrm{CO}_{2}$. U2OS and KHOS cells were placed in a medium containing DXR (Zhejiang Hisun Pharmaceutical CO., LTD,
Table 1 Correlation between has_circ_0004674 expression and clinicopathological characteristics of OS patients $(n=41)$

\begin{tabular}{|c|c|c|c|c|}
\hline \multirow[t]{2}{*}{$\begin{array}{l}\text { Pathological } \\
\text { characteristics }\end{array}$} & \multirow[t]{2}{*}{ Cases (n) } & \multicolumn{2}{|c|}{$\begin{array}{l}\text { Has_circ_0004674 } \\
\text { expression }\end{array}$} & \multirow[t]{2}{*}{$P$-value } \\
\hline & & High (20) & Low (21) & \\
\hline \multicolumn{5}{|l|}{ Gender } \\
\hline Male & 18 & 7 & 11 & 0.3499 \\
\hline Female & 23 & 13 & 10 & \\
\hline \multicolumn{5}{|l|}{ Age } \\
\hline$\geq 25$ & 16 & 7 & 9 & 0.7513 \\
\hline$<25$ & 25 & 13 & 12 & \\
\hline \multicolumn{5}{|l|}{ Enneking stage } \\
\hline$I+\| A$ & 13 & 3 & 10 & $0.0431^{*}$ \\
\hline$\|B+\|$ & 28 & 17 & 11 & \\
\hline \multicolumn{5}{|l|}{ Location } \\
\hline Distal of femur & 18 & 10 & 8 & 0.1956 \\
\hline Proximal of tibia & 14 & 8 & 6 & \\
\hline Other & 9 & 2 & 7 & \\
\hline \multicolumn{5}{|l|}{ Chemoresistant } \\
\hline Yes & 23 & 8 & 15 & $0.0427^{*}$ \\
\hline No & 18 & 12 & 6 & \\
\hline \multicolumn{5}{|l|}{ Type of disease } \\
\hline P53 mutation & 17 & 10 & 7 & 0.1112 \\
\hline Rb1 mutation & 14 & 8 & 6 & \\
\hline Other & 10 & 2 & 8 & \\
\hline
\end{tabular}

${ }^{*} P<0.05$

Taizhou, China), and the concentration of DXR was gradually increased from $2.5 \mathrm{ng} / \mathrm{mL}$ to $1 \mu \mathrm{g} / \mathrm{mL}$ over a period of 6 months as the previously reports [15]. After multiple screenings, DXR-resistant OS cell lines (U2OS_R and KHOS_R) were isolated.

Hsa_circ_0004674 small interference RNA (siRNA) or lentiviral short hairpin RNA (shRNA)

Table 2 The primer sequences in this study

\begin{tabular}{|c|c|}
\hline Gene & Sequences $\left(5^{\prime}-3^{\prime}\right)$ \\
\hline \multirow[t]{2}{*}{ hsa_circ_0004674 } & F: GCTTCCAGGTTGATGCCTTTG \\
\hline & R:TCCAGGAAGGTTGAAAGAATCCA \\
\hline \multirow[t]{2}{*}{ ADAM22 } & F: GAAGACGAAAGTCGGCACGA \\
\hline & R:TGAATGACGTTCCAAAGGCAT \\
\hline \multirow[t]{2}{*}{ miR-342-3p } & F: GGGTCTCACACAGAAATCGC \\
\hline & R: CAGTGCGTGTCGTGGAGT \\
\hline \multirow[t]{2}{*}{ FBN1 } & F:TTTAGCGTCCTACACGAGCC \\
\hline & R: CCATCCAGGGCAACAGTAAGC \\
\hline \multirow[t]{2}{*}{$\beta$-actin } & F: CTTCGCGGGCGACGAT \\
\hline & R: CCACATAGGAATCCTTCTGACC \\
\hline \multirow[t]{2}{*}{ U6 } & F: GCTTCGGCAGCACATATACTAAAAT \\
\hline & R: CGCTTCACGAATTTGCGTGTCAT \\
\hline
\end{tabular}


(si-hsa_circ_0004674\#1/\#2/\#3 or sh-hsa_circ_0004674), miR-342-3p mimic or inhibitor, pcDNA fibrillin-1 (FBN1) overexpression plasmid (pcDNA-FBN1), and negative controls (si-NC, sh-NC, mimic NC, inhibitor NC and pcDNA) were synthesized from RiboBio (Guangzhou, China). U2OS_R and KHOS_R cells were seeded into 6 -well plates $\left(4 \times 10^{5}\right.$ cells per well $)$ and incubated and incubated overnight at $37^{\circ} \mathrm{C}$. After the cells reached 50-60\% confluences, the above oligonucleotides $(40 \mathrm{nM})$ and vectors $(2 \mu \mathrm{g})$ were transfected into cells using Lipofectamine 3000 reagent (Invitrogen). Then, the cells were collected for function experiments after transfection for $48 \mathrm{~h}$.

\section{qRT-PCR}

RNA was extracted by TRIzol reagent (Invitrogen) and cDNA was obtained using BeyoRT ${ }^{\mathrm{TM}}$ II cDNA Synthesis Kit (Beyotime, Shanghai, China). QRT-PCR was performed by SYBR Green PCR Kit (Qiagen, Duesseldorf, Germany). Relative expression was normalized using $\beta$-actin or U6 and expressed using the $2^{-\Delta \Delta C T}$ method. Primer sequences were shown in Table 2.

\section{Identification of the circuRNA}

Genomic DNA (gDNA) was extracted from U2OS_R and KHOS_R cells using PureLink Genomic DNA Mini Kit (Invitrogen). After amplified with convergent primers and divergent primers using cDNA and gDNA, the PCR products of hsa_circ_0004674, $\beta$-actin and ADAM22 were detected by agarose gel electrophoresis. In RNase $\mathrm{R}$ assay, the extracted RNAs from U2OS_R and KHOS_R cells were incubated with or without RNase $\mathrm{R}$, and then the RNA was used for measuring hsa_circ_0004674 expression and linear ADAM22 expression.

\section{Cell counting kit 8 (CCK8) assay}

U2OS_R and KHOS_R cells were seeded into 96 well plates $\left(2 \times 10^{3}\right.$ cells/well). Freshly medium containing different concentrations of DXR was added into cells and

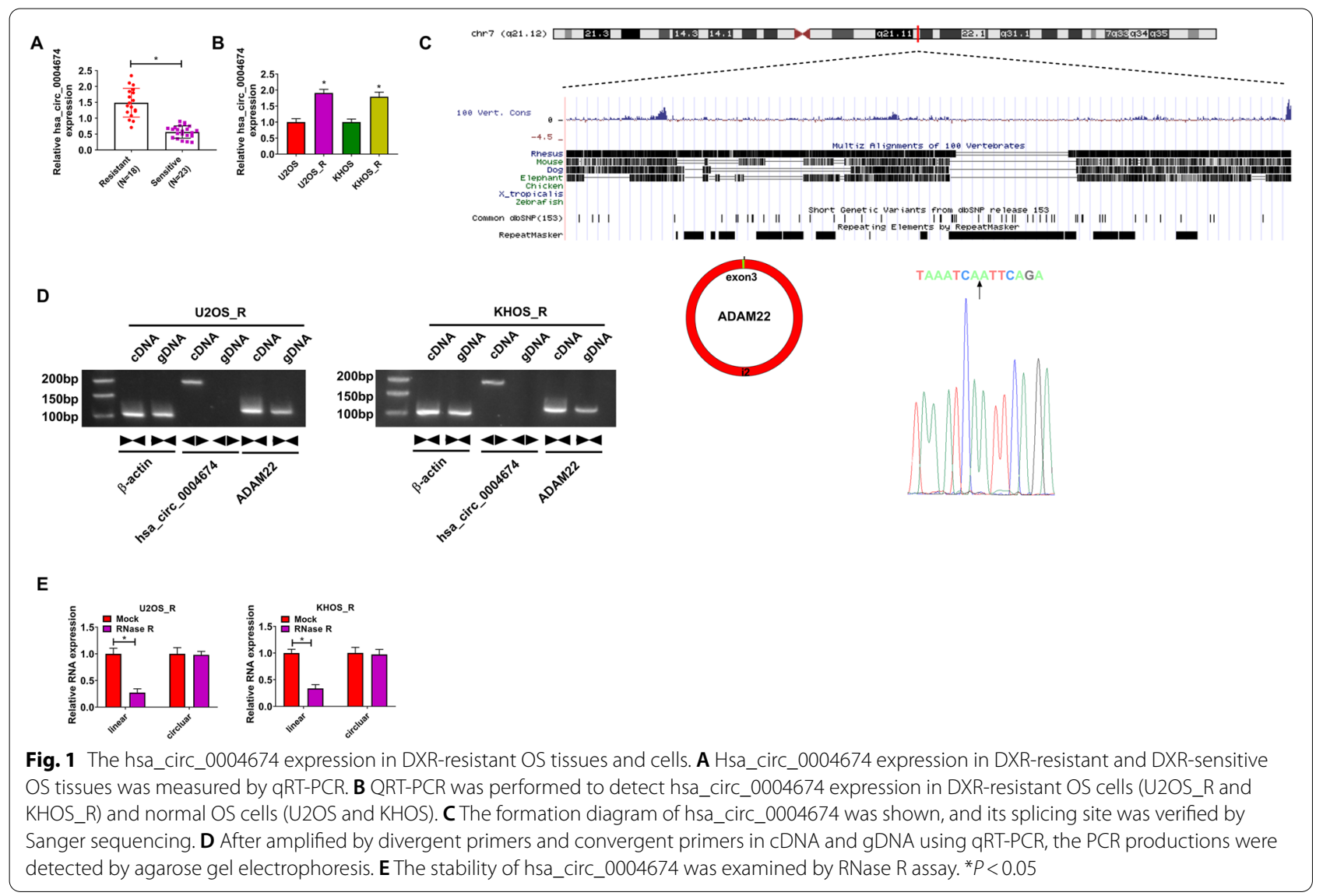


cultured for $48 \mathrm{~h}$. After that, CCK8 solution (Beyotime) was added into each well and incubated for $4 \mathrm{~h}$. Cell viability was measured at $450 \mathrm{~nm}$ to calculate $\mathrm{IC}_{50}$.

\section{Colony formation assay}

U2OS_R and KHOS_R cells were seeded in 6-well plates (250 cells/well) and cultured for 14 days. The colonies were stained by crystal violet, and its number was counted under microscope.

\section{Flow cytometry}

After transfection for 48 h, U2OS_R and KHOS_R cells were collected and then centrifuged at $1,000 \mathrm{rpm}$ for $5 \mathrm{~min}$. According to the instructions of Cell Cycle Analysis Kit (Beyotime), the cell suspensions $\left(2 \times 10^{6}\right.$ cells) were fixed with $70 \%$ ethanol and stained with RNase A and PI mixtures for $30 \mathrm{~min}$. For detecting cell apoptosis, the cell suspensions $\left(1 \times 10^{6}\right.$ cells $)$ were suspended with binding buffer and stained with Annexin V-FITC (Beyotime) and PI for $15 \mathrm{~min}$. Under flow cytometer, cell cycle process and apoptosis were analyzed using CellQuest software.

\section{Western blot (WB) analysis}

Protein was extracted by RIPA Lysis buffer (Sangon, Shanghai, China), subjected to SDS-PAGE gel, and transferred to PVDF membranes. The membranes were incubated with anti-p-glycoprotein (anti-p-gp, 1:500, Bioss, Beijing, China), anti-myeloid cell leukemia-1 (anti-MCL-1, 1:1,000, Bioss), anti-FBN1 (1:1,000, Bioss), anti- $\beta$-catenin (1:2,000, Bioss), anti-c-Myc $(1: 1,000$, Bioss $)$ or anti- $\beta$-actin (1:2,000, Bioss). After incubating with Goat Anti-Rabbit IgG (1:20,000, Bioss), the signals were determined by Enhanced ECL Chemiluminescence Detection Kit (Vazyme, Nanjing, China).

\section{Dual-luciferase reporter assay}

The hsa_circ_0004674 or FBN1 3'UTR wt and mut fragments were cloned into the pmirGLO vector. $293 \mathrm{~T}$ cells were seeded into 24 -well plates at $1 \times 10^{5}$ per well. The vectors $(50 \mathrm{ng})$ were transfected into $293 \mathrm{~T}$ cells with miR-342-3p mimic $(20 \mu \mathrm{M})$ or mimic NC $(20 \mu \mathrm{M})$. After 48 h, Dual-luciferase Reporter Assay System (Promega, Madison, WI, USA) was applied to analyze the luciferase activity.

\section{RNA pull-down assay}

U2OS_R and KHOS_R cells were transfected with BiomiR-342-3p probe or Bio-NC probe for $48 \mathrm{~h}$. Then, the cells were lysed and cell lysates were incubated with magnetic beads (Invitrogen), and the bounded RNA was purified using TRIzol reagent. The enrichment of hsa_circ_0004674 and FBN1 was analyzed by qRT-PCR.

\section{In vivo experiments}

Animal experiments were approved by the Animal Ethic Committee of Jingmen No.1 People's Hospital and performed according to the Guide for the Care and Use of Laboratory Animals. KHOS_R cells were transfected with sh-NC or sh-hsa_circ_0004674 and then subcutaneously injected into the flank of BALB/c nude mice $\left(n=6\right.$ per group, $1 \times 10^{6}$ cells/mouse; Vital River, Beijing, China). When the tumor grew about $100 \mathrm{~mm}^{3}, 3$ mice in each group were intraperitoneally injected with $2 \mathrm{mg} / \mathrm{kg} / \mathrm{d}$ DXR, and the remaining mice were injected with the same amount of PBS (Beyotime). The tumor volume was determined by detecting tumor length and width every 4 days. 16 days later, the tumor was removed for weighting and measuring protein expression.

\section{Statistical analysis}

All data were presented as mean \pm SD. All experiment was performed in triplicate, and all independent experiments were set for 3 times to take the average value. GraphPad Prism 5.0 software was utilized for the analysis. Student's $t$-test or ANOVA was used for statistical analysis. Correlations were analyzed by Pearson's correlation coefficient. $P<0.05$ was considered as significant.

\section{Results}

Hsa_circ_0004674 was highly expressed in DXR-resistant OS tissues and cells

In DXR-resistant and DXR-sensitive OS tissues, we discovered that hsa_circ_0004674 expression was remarkably higher in DXR-resistant OS tissues (Fig. 1A). The correlation analysis between has_circ_0004674 expression and clinicopathological characteristics of OS patients showed that high expression of has_circ_0004674 was associated with Enneking stage and chemoresistance in OS patients (Table 1). In addition, hsa_circ_0004674 was upregulated in DXR-resistant OS cells (U2OS_R and KHOS_R) compared to U2OS and KHOS cells (Fig. 1B). Through analysis, we confirmed that hsa_circ_0004674 is formed by the back-splicing of ADAM22 gene, as confirmed by Sanger sequencing (Fig. 1C). After amplified by convergent primers and divergent primers, we found that hsa_circ_0004674 could be amplified by divergent primers in cDNA but not in gDNA, while linear RNA $\beta$-actin and ADAM22 could be amplified by convergent primers in both cDNA and gDNA (Fig. 1D), which suggested that hsa_circ_0004674 had circular structure. Using the RNase R assay, we confirmed that hsa_circ_0004674 could resist the digestion of RNase $\mathrm{R}$ compared to its linear mRNA ADAM22 (Fig. 1E). 
Hsa_circ_0004674 knockdown reduced OS DXR resistance To identify the function of hsa_circ_0004674 in OS chemoresistance, three siRNAs for hsa_circ_0004674 were transfected into U2OS_R and KHOS_R cells to silence hsa_circ_0004674 expression. The results suggested that all three siRNAs could significantly reduce the expression of hsa_circ_0004674, especially si-hsa_circ_0004674\#1 and si-hsa_circ_0004674\#2 had better effects (Fig. 2A), so both were chosen for functional research. The $\mathrm{IC}_{50}$ of U2OS_R and KHOS_R cells after hsa_circ_0004674 knockdown was obviously decreased (Fig. 2B). Moreover, hsa_circ_0004674 silencing inhibited the number of colonies in OS cells (Fig. 2C), and promoted the cell number in G0/G1 phase to inhibit the cell number in $S$ phase (Fig. 2D). These data indicated that OS cell proliferation was suppressed by hsa_circ_0004674 downregulation. In addition, hsa_circ_0004674 knockdown also promoted the apoptosis rate of U2OS_R and KHOS_R cells (Fig. 2E), and inhibited resistance markers p-gp and MCL-1 protein levels (Fig. 2F).

\section{Hsa_circ_0004674 sponged miR-342-3p}

In order to explore the potential mechanism of hsa circ_0004674, we conducted bioinformatics analysis. The analysis result of starBase software showed that miR-342-3p had binding sites for hsa_circ_0004674. Then, we constructed the hsa_circ_0004674 wt and hsa_ circ_0004674 mut vectors (Fig. 3A). MiR-342-3p mimic could specifically reduce the luciferase activity of hsa_ circ_0004674 wt vector without affecting that of the mut vector (Fig. 3B). In addition, RNA pull-down assay results concluded that hsa_circ_0004674 could be significantly enriched in the Bio-miR-342-3p probe compared to that in Bio-NC probe (Fig. 3C). These data confirmed the interaction between hsa_circ_0004674 and miR-342-3p. Furthermore, miR-342-3p was found to be downregulated in DXR-resistant OS tissues and cells (Fig. 3D-E), and was also negatively correlated with hsa_circ_0004674 expression in OS tissues (Fig. 3F).

\section{MiR-342-3p inhibitor abolished the regulation of hsa_ circ_0004674 silencing on OS DXR resistance}

Subsequently, we explored whether hsa_circ_0004674 regulated OS chemoresistance by miR-342-3p. The significant decrease in miR-342-3p expression confirmed the transfection effectiveness of miR-342-3p inhibitor (Fig. 4A). Then, si-hsa_circ_0004674\#1 and miR342-3p inhibitor were co-transfected into OS cells. The promotion effect of si-hsa_circ_0004674\#1 on miR-342-3p expression could be effectively restored by miR-342-3p inhibitor (Fig. 4B). CCK8 assay suggested that the $\mathrm{IC}_{50}$ reduced by hsa_circ_0004674 silencing could be recovered by miR-342-3p inhibitor (Fig. 4C). The suppressive effects of hsa_circ_0004674 knockdown on the number of colonies and cell cycle process in U2OS_R and KHOS_R cells also were overturned by miR-342-3p inhibitor (Fig. 4D-E). Additionally, miR-342-3p inhibitor also abolished the promotion effect of hsa_circ_0004674 knockdown on cell apoptosis rate (Fig. 4F), as well as the inhibitory effect on the protein levels of p-gp and MCL-1 in U2OS_R and KHOS_R cells (Fig. 4G). All results revealed that hsa circ_0004674 sponged miR-342-3p to regulate OS DXR resistance.

\section{FBN1 was targeted by miR-342-3p}

Similarly, we also used the Starbase software to predict the targets of miR-342-3p and the 3'UTR of FBN1 was discovered to have binding sites for miR-342-3p (Fig. 5A). MiR-342-3p overexpression could inhibit the luciferase activity of FBN1 3'UTR wt vector, while had not effect on that of the mut vector (Fig. 5B). And the enrichment of FBN1 in the Bio-miR-342-3p probe was higher than the control probe (Fig. 5C). Further, we found a significantly high expression of FBN1 in DXR-resistant OS tissues at the mRNA and protein levels (Fig. 5D-E). Compared to normal OS cells (U2OS and KHOS), FBN1 protein expression in DXR-resistant OS cells (U2OS_R and KHOS_R) was notably increased (Fig. 5F). Additionally, FBN1 expression was negatively correlated with miR342-3p expression in OS tissues (Fig. 5G).

\section{FBN1 reversed the regulation of miR-342-3p on OS DXR resistance}

To further investigate whether FBN1 participated in the regulation of miR-342-3p on OS DXR resistance, we performed rescue experiments. MiR-342-3p mimic could significantly promote miR-342-3p expression (Fig. 6A), and pcDNA-FBN1 could remarkably enhance the protein expression of FBN1 (Fig. 6B). After co-transfecting, we found that the decreasing effect of miR-342-3p mimic

\footnotetext{
(See figure on next page.)

Fig. 2 Hsa_circ_0004674 knockdown reduced OS DXR resistance. A The transfection efficiencies of three siRNAs for hsa_circ_0004674 were evaluated by detecting hsa_circ_0004674 expression using qRT-PCR in U2OS_R and KHOS_R cells. B-F U2OS_R and KHOS_R cells were transfected with si-NC, si-hsa_circ_0004674\#1 or si-hsa_circ_0004674\#2. B CCK8 assay was used to test the cell DXR resistance. Colony formation assay (C) and flow cytometry ( $\mathbf{D}-\mathbf{E})$ were performed to determine the number of colonies, cell cycle process and apoptosis rate. $\mathbf{F}$ The $\mathrm{p}$-gp and $\mathrm{MCL}-1 \mathrm{protein}$ levels were tested by WB analysis. ${ }^{*} P<0.05$
} 

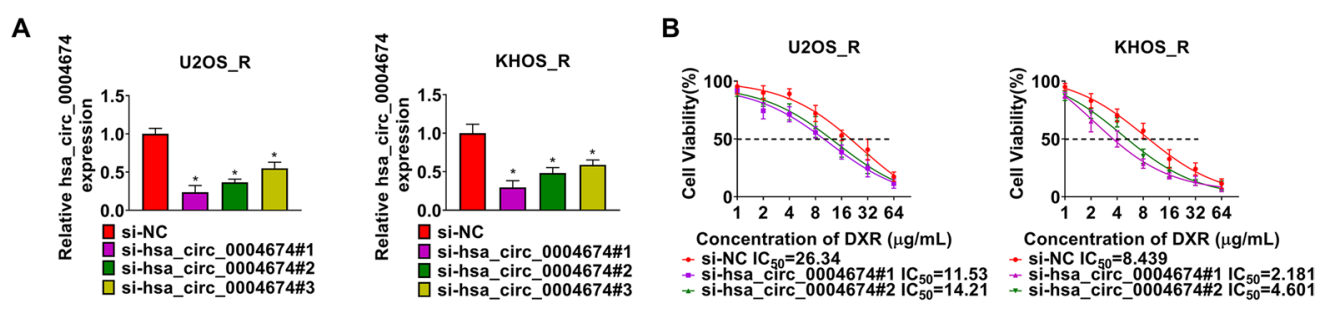

C
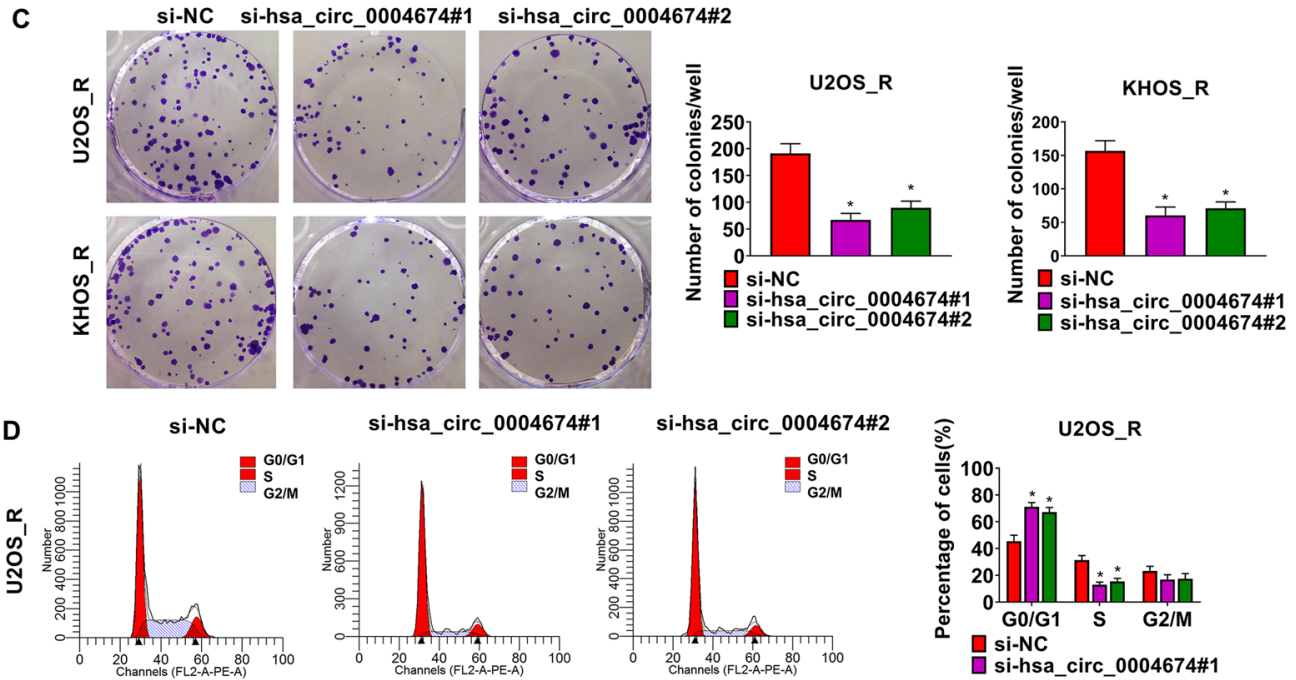

si-hsa_circ_0004674\#1

si-hsa_circ_0004674\#2
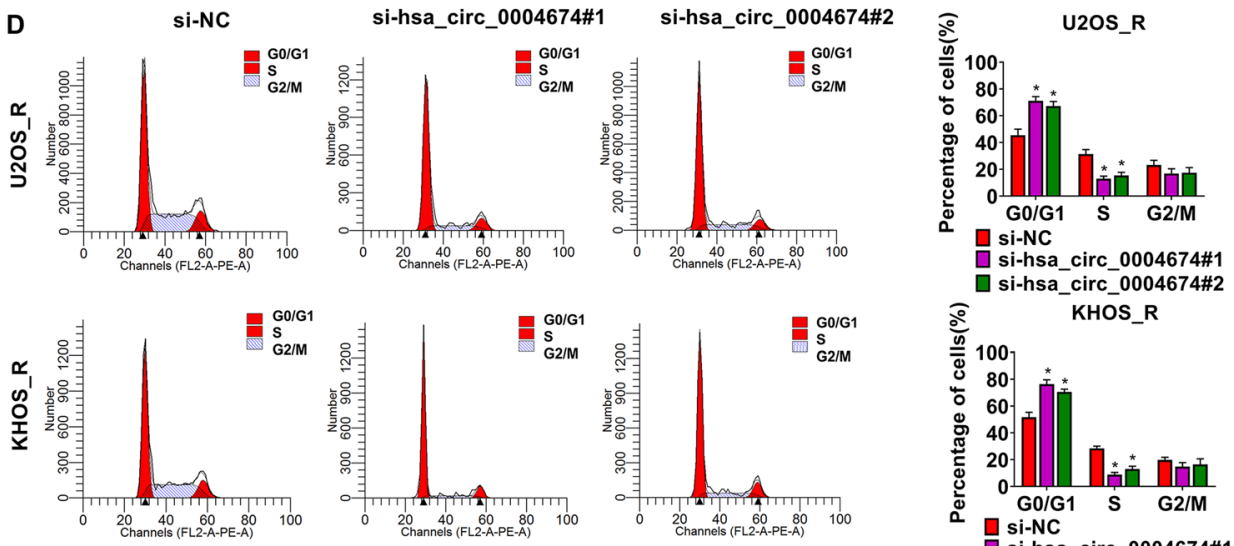

E si-NC si-hsa_circ_0004674\#1 si-hsa_circ_0004674\#2
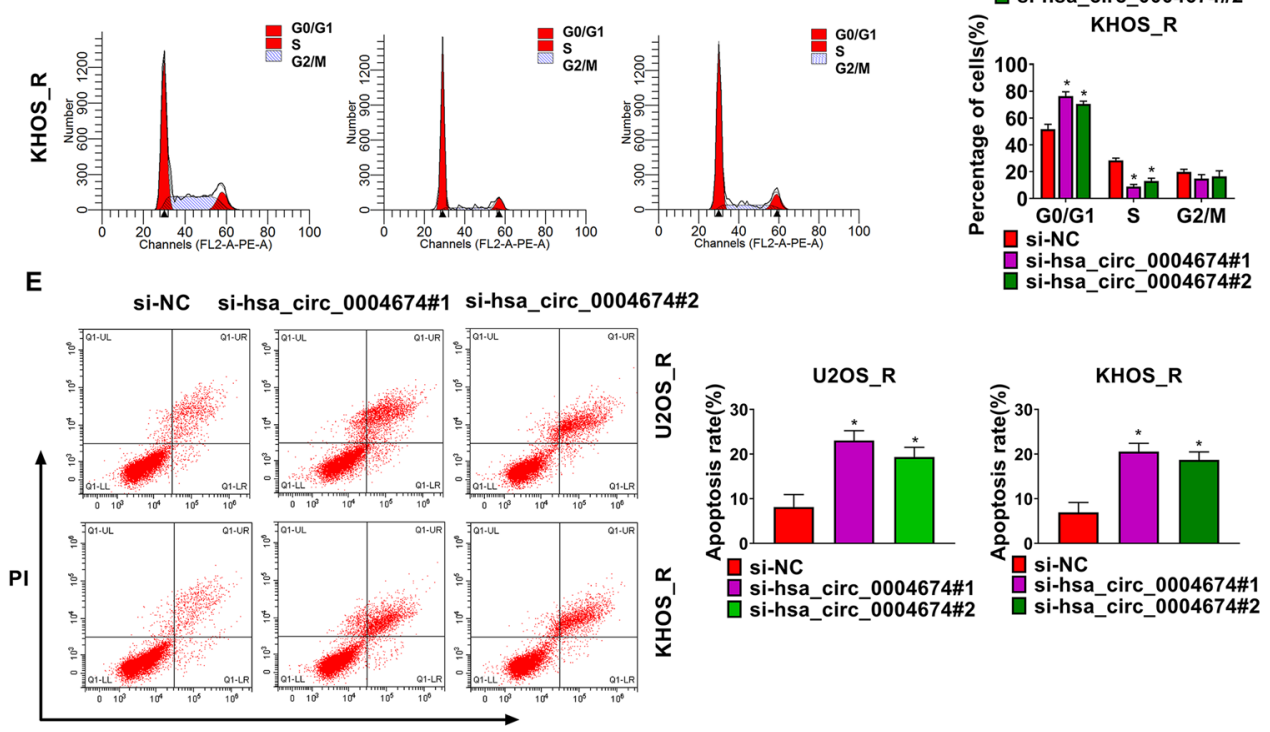

Annexin FITC
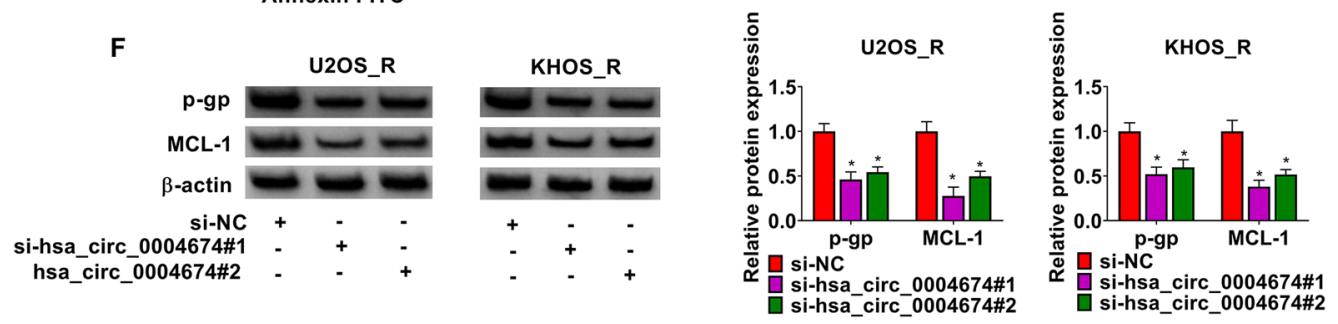

Fig. 2 (See legend on previous page.) 


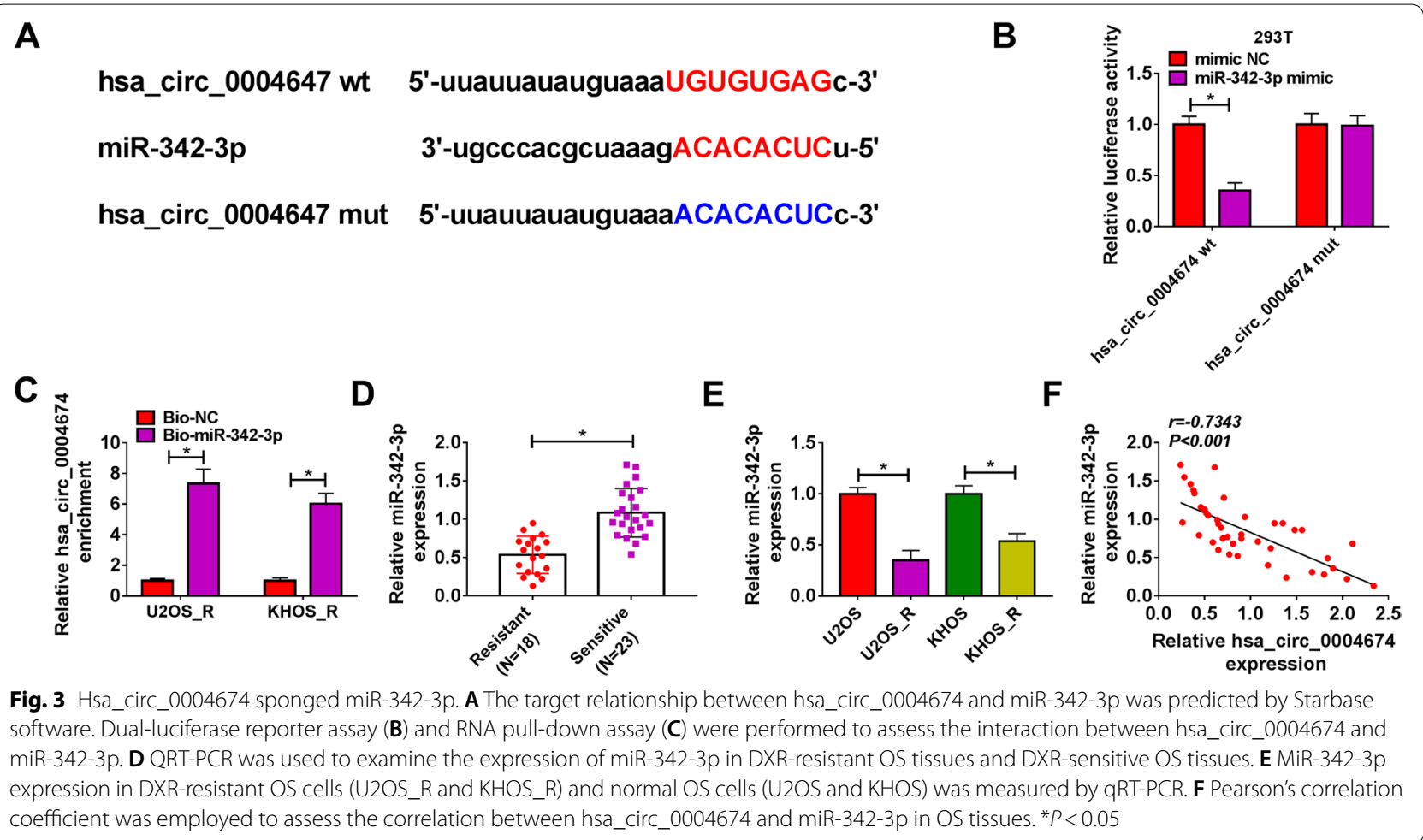

on FBN1 protein expression could be abolished by overexpressing FBN1 (Fig. 6C). MiR-342-3p suppressed the $\mathrm{IC}_{50}$, and this effect could be recovered by the addition of pcDNA-FBN1 (Fig. 6D). Meanwhile, miR-342-3p mimic hindered the number of colonies and induced cell cycle arrest, while FBN1 overexpression also could overturn these effects (Fig. 6E-F). Furthermore, overexpressed FBN1 also abolished the promotion effect of miR-342-3p on apoptosis rate and the inhibitory effect on the protein levels of p-gp and MCL-1 in U2OS_R and KHOS_R cells (Fig. 6G$H)$. Our results illuminated that miR-342-3p restrained the DXR resistance of OS cells by targeting FBN1.

\section{The hsa_circ_0004674/miR-342-3p/FBN1 axis regulated the activity of Wnt/ $\beta$-catenin pathway}

Wnt $/ \beta$-catenin pathway is involved in cancer cell chemoresistance $[16,17]$. To evaluate whether the hsa circ_0004674//miR-342-3p/FBN1 axis also regulated the activity of the Wnt/ $\beta$-catenin pathway, we detected $\beta$-catenin and $c-M y c$ protein expression. As shown in Fig. 7A-B, WB analysis revealed that hsa_circ_0004674 silencing could obviously inhibit $\beta$-catenin and c-Myc protein levels. However, the addition of miR-342-3p inhibitor or pcDNA-FBN1 reversed the suppressive effect of silenced-hsa_circ_0004674 on $\beta$-catenin and c-Myc protein levels. These data revealed that hsa circ_0004674 could enhance Wnt/ $\beta$-catenin pathway activity via regulating the $\mathrm{miR}-342-3 \mathrm{p} / \mathrm{FBN} 1$ axis.
Interference of hsa_circ_0004674 enhanced DXR sensitive of OS tumors

In addition, we conducted animal experiments to assess the role of hsa_circ_0004674 in the DXR resistance of OS tumors. Through measuring the tumor weight and tumor volume of OS, we found that hsa_circ_0004674 silencing or DXR treatment could markedly reduce the tumor volume and weight (Fig. 8A-B). In the DXR treatment group, hsa_circ_0004674 knockdown had a more obvious inhibitory effect on tumor growth, suggesting that hsa_circ_0004674 silencing could promote the DXR sensitive of OS tumors (Fig. 8A-B). Additionally, the protein expression levels of resistance markers (p-gp and MCL-1) and Wnt/ $\beta$-catenin pathway markers ( $\beta$-catenin and $\mathrm{c}-\mathrm{Myc}$ ) in OS tumor tissues were significantly decreased by hsa_circ_0004674 knockdown and DXR treatment (Fig. 8C).

\section{Discussion}

DXR is an anti-tumor antibiotic, which is often used as the treatment of most malignant tumors in clinic, including OS, liver cancer, gastric cancer, and breast cancer $[18,19]$. At present, many circRNAs have been found to participate in regulating OS chemoresistance. For example, hsa_circ_0000073 could promote the methotrexate resistance of OS [20], and circPVT1 regulated the DXR and cisplatin resistance of OS [21]. Hsa_circ_0004674 is a newly 


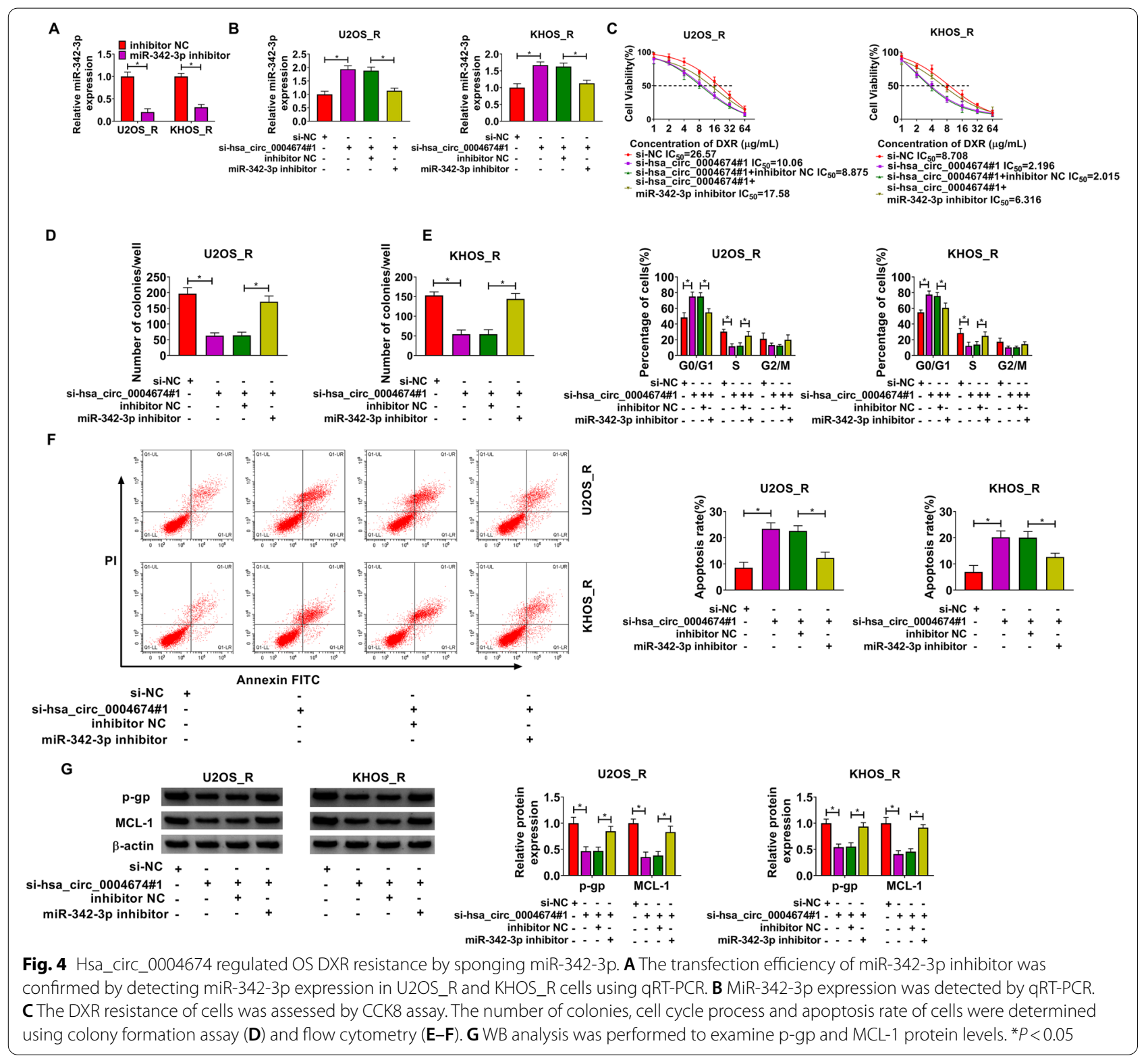

discovered circRNA. Consistent with the results of previous analyses [14], we discovered a high hsa circ_0004674 expression in OS DXR-resistant cells and tissues. Hsa_circ_0004674 knockdown could lead to the decreased DXR resistance, reduced proliferation and increased apoptosis of OS cells. Silencing of hsa_circ_0004674 enhanced the sensitivity of OS tumors to DXR. These data indicated that the increased hsa_circ_0004674 expression was essential for maintaining the DXR resistance of OS, suggesting that hsa_circ_0004674 knockdown might be an effective way to treat OS chemoresistance.
MiR-342-3p is considered to be a suppressor in many tumors, including cervical cancer [22], hepatocellular carcinoma [23] and glioma [24]. Importantly, low miR-342-3p expression was related to tumor chemoresistance. MiR-342-3p could repress colorectal cancer chemoresistance [25], and could act synergistically with miR-205-5p to reduce tumor chemoresistance by inhibiting E2F1 [26]. MiR-342-3p had an obvious tendency of low expression in OS, and has been proved to inhibit the progression and promote the radiosensitivity of OS [27-29]. Our study discovered that miR342-3p was underexpressed in the DXR-resistant OS 


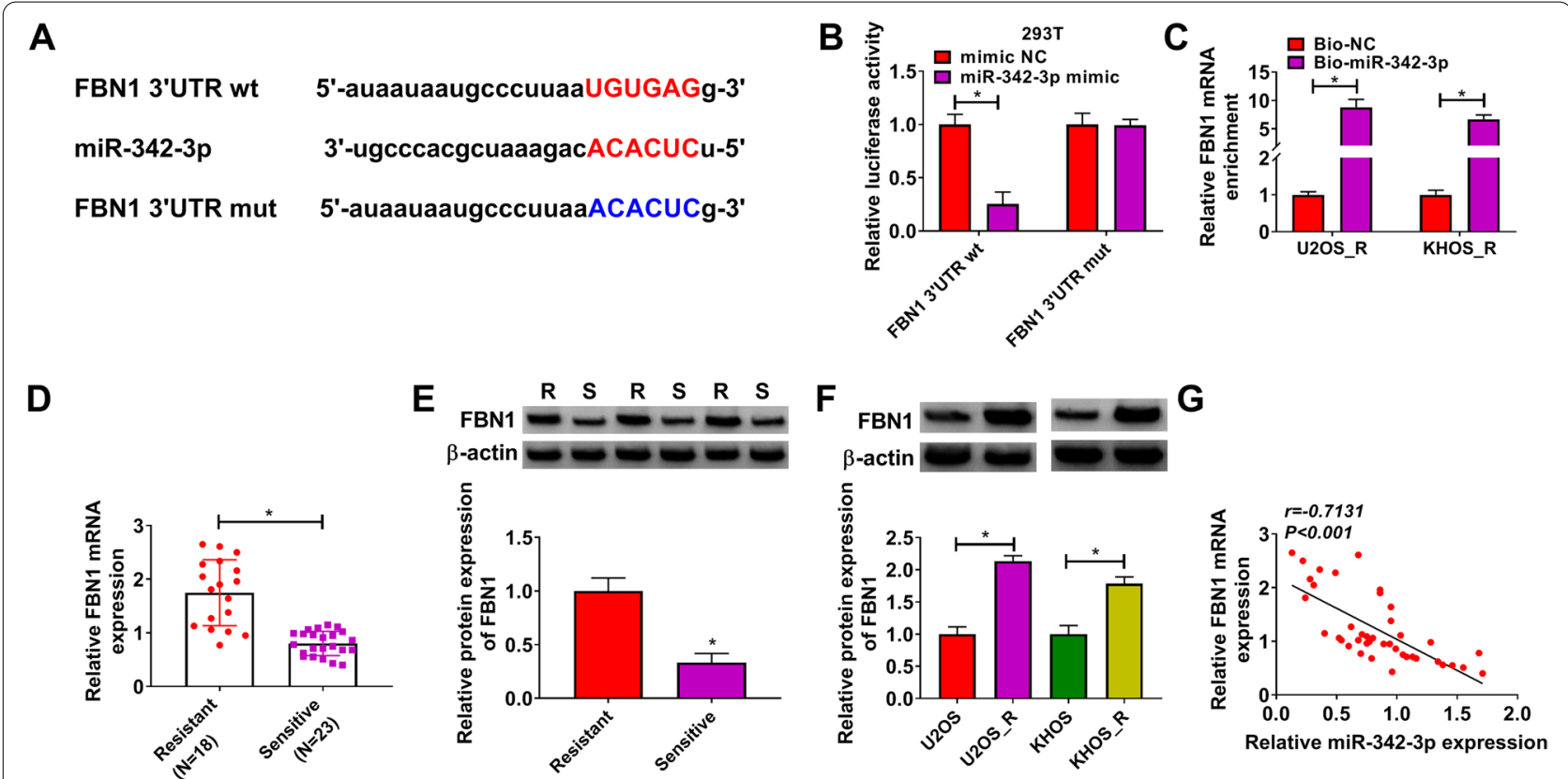

Fig. 5 FBN1 was targeted by miR-342-3p. A The binding sites between miR-342-3p and FBN1 3'UTR was predicted by Starbase software. The interaction between miR-342-3p and FBN1 was detected by dual-luciferase reporter assay (B) and RNA pull-down assay (C). D-E FBN1 mRNA and protein expression levels in DXR-resistant OS tissues and DXR-sensitive OS tissues were measured by qRT-PCR and WB analysis. F The FBN1 protein expression in DXR-resistant OS cells (U2OS_R and KHOS_R) and normal OS cells (U2OS and KHOS) was detected by WB analysis. G The correlation between miR-342-3p and FBN1 in OS tissues was analyzed by Pearson's correlation coefficient. ${ }^{*} P<0.05$

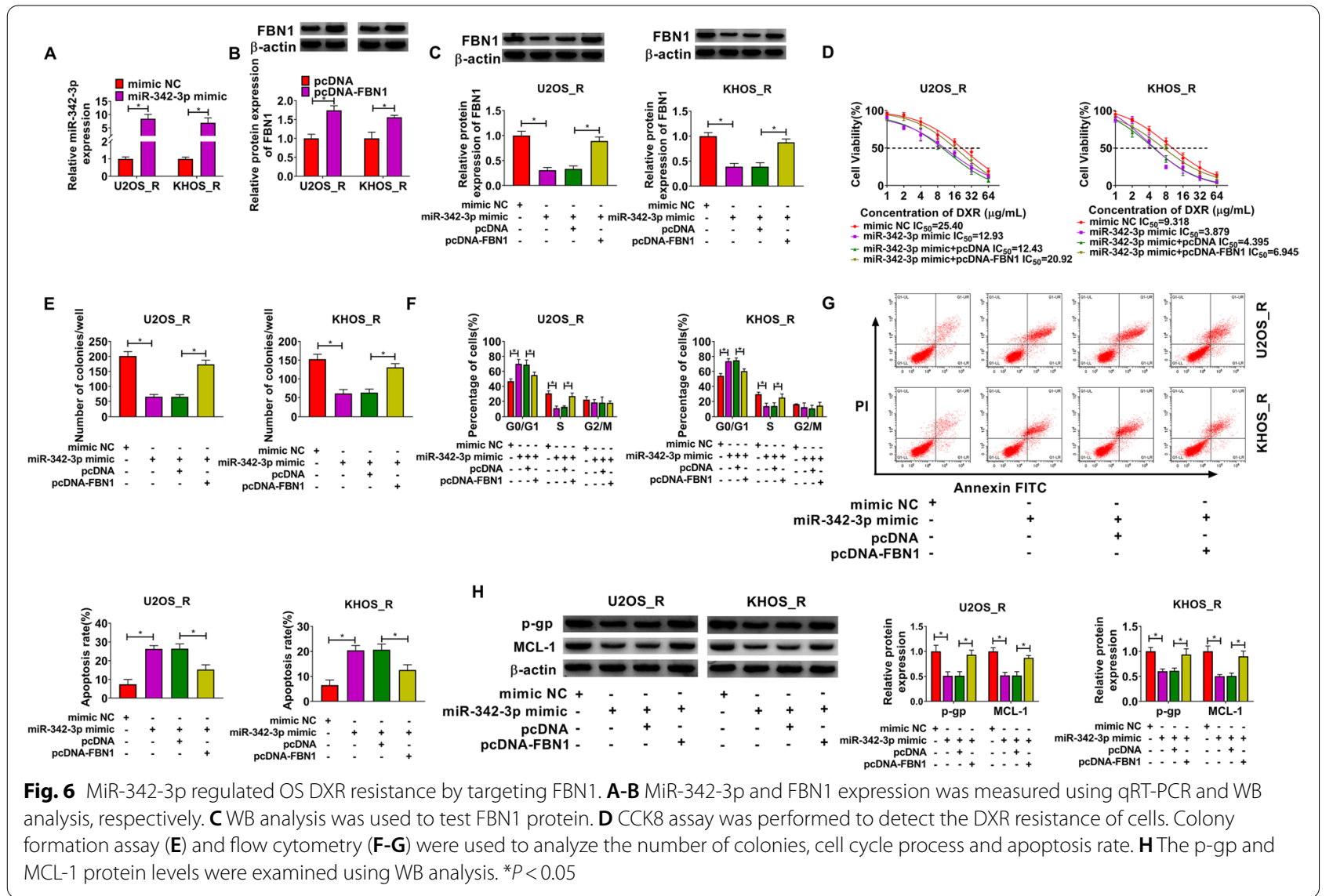



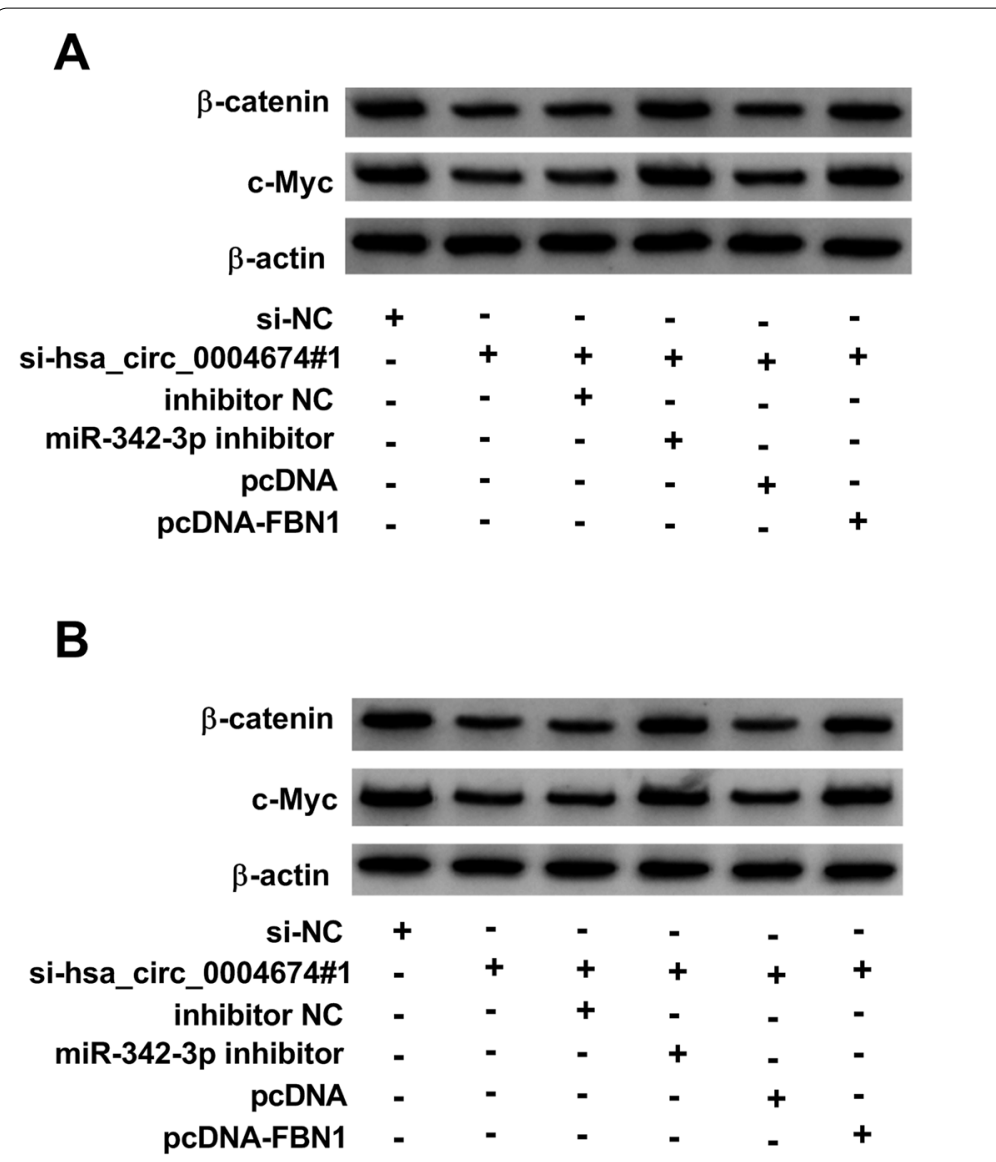
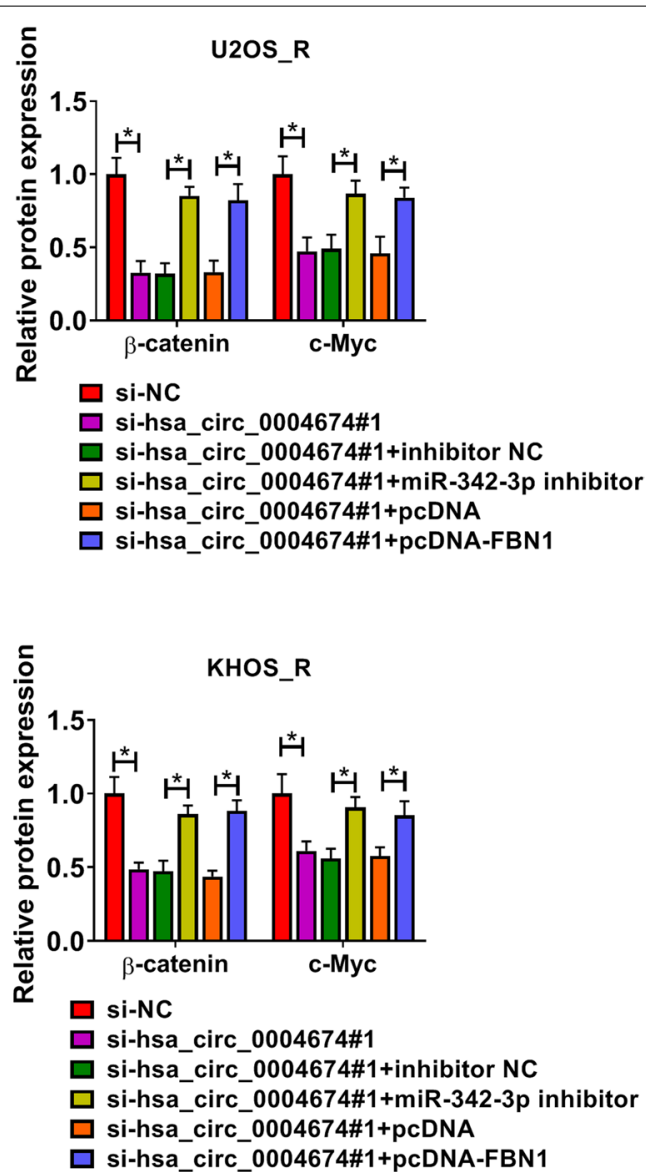

Fig. 7 The hsa_circ_0004674/miR-342-3p/FBN1 axis regulated Wnt/ $\beta$-catenin pathway activity. A-B The $\beta$-catenin and c-Myc protein expression levels were detected by WB analysis in U2OS_R and KHOS_R cells. ${ }^{*} P<0.05$ tissues and cells, and it also hindered OS DXR resistance. The reversal effect of anti-miR-342-3p on sihsa_circ_0004674 suggested that hsa_circ_0004674 regulated the DXR resistance of OS via targeting miR-342-3p.

Further experiments revealed that miR-342-3p targeted FBN1. FBN1 is the main component of extracellular matrix microfibrils, which can maintain the integrity and normal function of connective tissue [30, 31]. In addition to Marfan syndrome caused by FBN1 mutations, many studies have also reported that abnormal FBN1 expression is related to tumor malignant phenotype, including ovarian cancer and papillary thyroid carcinoma [32, 33]. Liu et al. showed that FNB1 was upregulated in OS, and its knockdown could inhibit OS tumorigenesis [34]. Therefore, FNB1 also had a positive role in OS progression. Our data also revealed that miR-342-3p promoted the DXR resistance by targeting FBN1.
Wnt $/ \beta$-catenin is one of the important signaling pathways that mediate embryonic development and cell biological functions, and it is abnormally activated in a variety of human diseases [35]. Studies have shown that the activity of the $\mathrm{Wnt} / \beta$-catenin pathway is significantly up-regulated in the malignant progression of many cancers [36]. Targeted inhibition of $\mathrm{Wnt} / \beta$ catenin pathway is considered to be an effective way of cancer treatment, including OS $[37,38]$. In the related studies of OS, activation of $\mathrm{Wnt} / \beta$-catenin pathway has been found to be related to chemoresistance of OS, and inhibition of $\mathrm{Wnt} / \beta$-catenin pathway has been proved to improve the chemotherapy sensitivity of OS $[39,40]$. In this, we discovered that silencing of hsa_circ_0004674 could inhibit the activity of the Wnt/ $\beta$-catenin pathway. Further analysis showed that hsa_circ_0004674 positively regulated $\mathrm{Wnt} / \beta$-catenin pathway activity through the miR-342-3p/FBN1 axis. These results are a new discovery for us. 


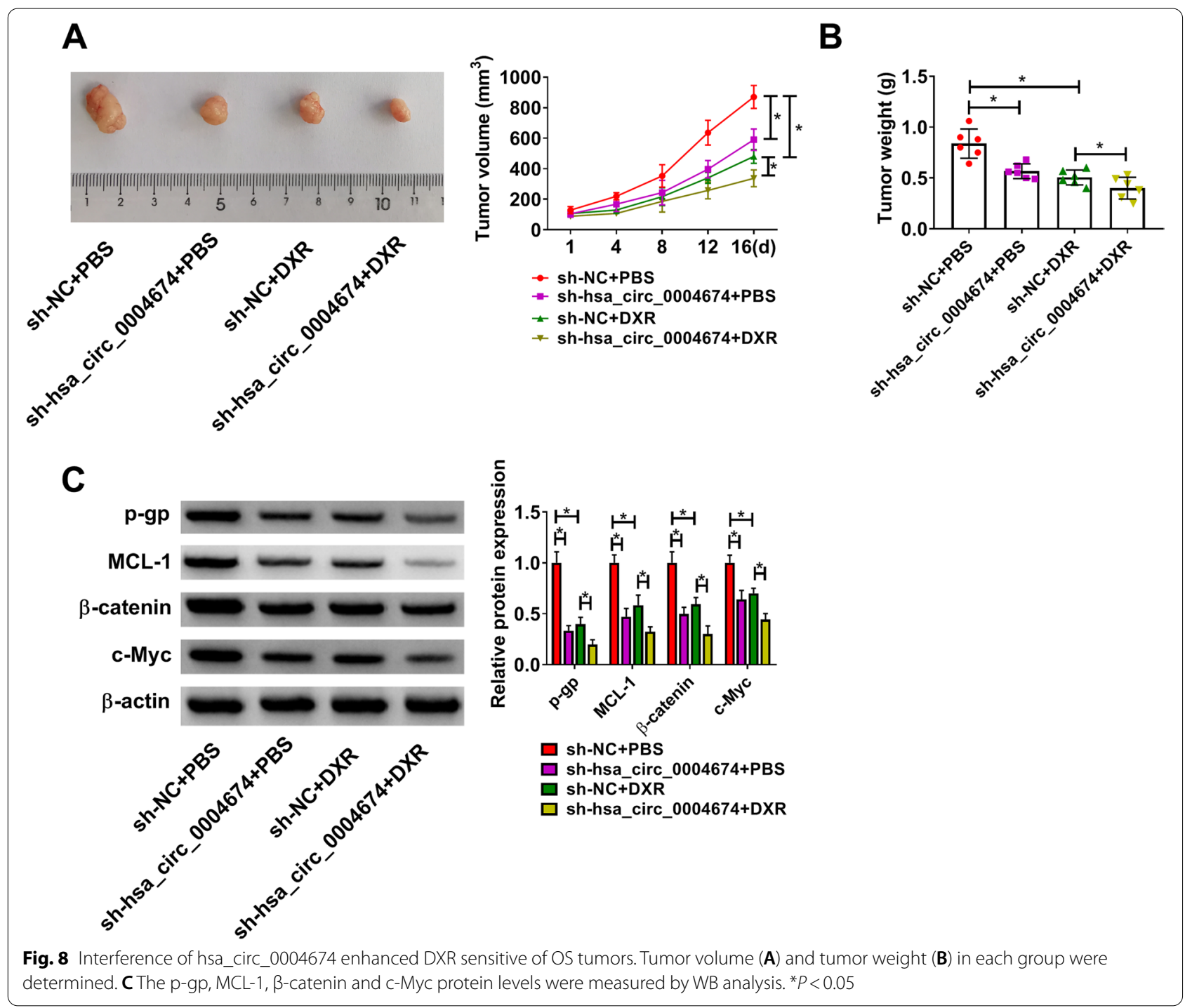

\section{Conclusion}

In conclusion, our experiment confirmed the function of hsa_circ_0004674 in OS chemoresistance for the first time. We found that hsa circ 0004674 could facilitate OS chemoresistance via miR-342-3p/FBN1 axis to activate the Wnt/ $\beta$-catenin pathway. Our research provided a theoretical basis for overcoming the chemoresistance of OS.

\section{Abbreviations}

circRNA: Circular RNA; OS: Osteosarcoma; FBN1: Fibrillin-1; CCK8: Cell counting kit 8; DXR: Doxorubicin; WB: Western blot.

\section{Acknowledgements}

None

\section{Authors' contributions}

Yanghua Li designed and performed the research; Juan Bai and Juan Bai analyzed the data; Yumei Bai wrote the manuscript. All authors read and approved the final manuscript.
Funding

None.

Availability of data and materials

Not applicable.

\section{Declarations}

Ethics approval and consent to participate

Written informed consents were obtained from all participants and this study was permitted by the Ethics Committee of Jingmen No.1 People's Hospital.

Consent for publication

Not applicable.

\section{Competing interests}

The authors declare that they have no conflict of interest.

Received: 29 April 2021 Accepted: 24 July 2021

Published online: 18 August 2021 


\section{References}

1. Ritter J, Bielack SS. Osteosarcoma. Ann Oncol. 2010;21(Suppl 7):vii320-5.

2. Simpson E, Brown HL. Understanding osteosarcomas. JAAPA. 2018;31(8):15-9

3. Zhu W, Zhu L, Bao Y, Zhong X, Chen Y, Wu Q. Clinical evaluation of neoadjuvant chemotherapy for osteosarcoma. J Buon. 2019;24(3):1181-5.

4. Bacci G, Ferrari S, Lari S, Mercuri M, Donati D, Longhi A, et al. Osteosarcoma of the limb. Amputation or limb salvage in patients treated by neoadjuvant chemotherapy. J Bone Joint Surg Br. 2002;84(1):88-92.

5. d'Amato TA, Landreneau RJ, Ricketts W, Huang W, Parker R, Mechetner $E$, et al. Chemotherapy resistance and oncogene expression in nonsmall cell lung cancer. J Thorac Cardiovasc Surg. 2007;133(2):352-63.

6. Hughes D, Andersson DI. Evolutionary consequences of drug resistance: shared principles across diverse targets and organisms. Nat Rev Genet. 2015;16(8):459-71.

7. Jiang WD, Yuan PC. Molecular network-based identification of competing endogenous RNAs in bladder cancer. PLoS One. 2019;14(8):e0220118.

8. Zhong Y, Du Y, Yang X, Mo Y, Fan C, Xiong F, et al. Circular RNAs function as ceRNAs to regulate and control human cancer progression. Mol Cancer. 2018;17(1):79

9. Zhao X, Cai Y, Xu J. Circular RNAs: biogenesis, mechanism, and function in human cancers. Int J Mol Sci. 2019;20(16):3926.

10. Ng WL, Mohd Mohidin TB, Shukla K. Functional role of circular RNAs in cancer development and progression. RNA Biol. 2018;15(8):995-1005.

11. Zhang Z, Yu X, Zhou B, Zhang J, Chang J. Circular RNA circ_0026359 enhances cisplatin resistance in gastric cancer via targeting miR-1200/ POLD4 pathway. Biomed Res Int. 2020;2020:5103272.

12 Yin H, Cui X. Knockdown of circHIPK3 Facilitates Temozolomide Sensitivity in Glioma by Regulating Cellular Behaviors Through miR-524-5p/KIF2AMediated PI3K/AKT Pathway. Cancer Biother Radiopharm. 2020. https:// doi.org/10.1089/cbr.2020.3575.

13. Liang $Y$, Song $X$, Li Y, Su P, Han D, Ma T, et al. circKDM4C suppresses tumor progression and attenuates doxorubicin resistance by regulating miR548p/PBLD axis in breast cancer. Oncogene. 2019;38(42):6850-66.

14. Kun-Peng Z, Xiao-Long M, Lei Z, Chun-Lin Z, Jian-Ping H, Tai-Cheng Z. Screening circular RNA related to chemotherapeutic resistance in osteosarcoma by RNA sequencing. Epigenomics. 2018;10(10):1327-46.

15 Hu T, Fei Z, Su H, Xie R, Chen L. Polydatin inhibits proliferation and promotes apoptosis of doxorubicin-resistant osteosarcoma through LncRNA TUG1 mediated suppression of Akt signaling. Toxicol Appl Pharmacol. 2019:371:55-62

16. Han P, Li JW, Zhang BM, Lv JC, Li YM, Gu XY, et al. The IncRNA CRNDE promotes colorectal cancer cell proliferation and chemoresistance via miR-181a-5p-mediated regulation of Wnt/beta-catenin signaling. Mo Cancer. 2017;16(1):9

17. Lin Z, Sun L, Xie S, Zhang S, Fan S, Li Q, et al. Chemotherapy-induced long non-coding RNA 1 promotes metastasis and chemo-resistance of TSCC via the Wnt/beta-catenin signaling pathway. Mol Ther. 2018;26(6):1494-508.

18. Rivankar S. An overview of doxorubicin formulations in cancer therapy. Cancer Res Ther. 2014;10(4):853-8.

19. Tacar O, Sriamornsak P, Dass CR. Doxorubicin: an update on anticancer molecular action, toxicity and novel drug delivery systems. J Pharm Pharmacol. 2013;65(2):157-70.

20. Li X, Liu Y, Zhang X, Shen J, Xu R, Liu Y, et al. Circular RNA hsa_ circ_0000073 contributes to osteosarcoma cell proliferation, migration, invasion and methotrexate resistance by sponging miR-145-5p and miR151-3p and upregulating NRAS. Aging. 2020;12(14):14157-73.

21. Kun-Peng Z, Xiao-Long M, Chun-Lin Z. Overexpressed circPVT1, a potential new circular RNA biomarker, contributes to doxorubicin and cisplatin resistance of osteosarcoma cells by regulating $A B C B 1$. Int J Biol Sci. 2018;14(3):321-30.

22. Peng X, Gao J, Cai C, Zhang Y. LncRNA LINC01503 aggravates the progression of cervical cancer through sponging miR-342-3p to mediate FXYD3 expression. Biosci Rep. 2020:40(6):BSR20193371.

23. Komoll RM, Hu Q, Olarewaju O, von Dohlen L, Yuan Q, Xie Y, et al. MicroRNA-342-3p is a potent tumour suppressor in hepatocellular carcinoma. J Hepatol. 2020;74:122-34.
24. Cheng G, Zheng J, Wang L. LncRNA SNHG7 promotes glioma cells viability, migration and invasion by regulating miR-342-3p/AKT2 Axis. Int J Neurosci. 2020;1-14.

25. Zhang PF, Wu J, Wu Y, Huang W, Liu M, Dong ZR, et al. The IncRNA SCARNA2 mediates colorectal cancer chemoresistance through a conserved microRNA-342-3p target sequence. J Cell Physiol. 2019;234(7):10157-65.

26. Lai X, Gupta SK, Schmitz U, Marquardt S, Knoll S, Spitschak A, et al. MiR-205-5p and miR-342-3p cooperate in the repression of the E2F1 transcription factor in the context of anticancer chemotherapy resistance. Theranostics. 2018;8(4):1106-20.

27. Chen H, Wahafu P, Wang L, Chen X. LncRNA LINC00313 knockdown inhibits tumorigenesis and metastasis in human osteosarcoma by upregulating FOSL2 through sponging miR-342-3p. Yonsei Med J. 2020;61(5):359-70.

28. Zhang S, Liu L, Lv Z, Li Q, Gong W, Wu H. MicroRNA-342-3p Inhibits the proliferation, migration, and invasion of osteosarcoma cells by targeting astrocyte-elevated gene-1 (AEG-1). Oncol Res. 2017;25(9):1505-15.

29 He P, Xu YQ, Wang ZJ, Sheng B. LncRNA LINC00210 regulated radiosensitivity of osteosarcoma cells via miR-342-3p/GFRA1 axis. J Clin Lab Anal. 2020;34:e23540.

30. Sakai LY, Keene DR, Renard M, De Backer J. FBN1: The disease-causing gene for Marfan syndrome and other genetic disorders. Gene. 2016;591(1):279-91.

31 Sakai LY, Keene DR. Fibrillin protein pleiotropy: Acromelic dysplasias. Matrix Biol. 2019;80:6-13.

32. Wang Z, Liu Y, Lu L, Yang L, Yin S, Wang Y, et al. Fibrillin-1, induced by Aurora-A but inhibited by BRCA2, promotes ovarian cancer metastasis. Oncotarget. 2015;6(9):6670-83.

33 Ma X, Wei J, Zhang L, Deng D, Liu L, Mei X, et al. miR-486-5p inhibits cel growth of papillary thyroid carcinoma by targeting fibrillin-1. Biomed Pharmacother. 2016;80:220-6.

34. Liu W, Liu P, Gao H, Wang X, Yan M. Long non-coding RNA PGM5-AS1 promotes epithelial-mesenchymal transition, invasion and metastasis of osteosarcoma cells by impairing miR-140-5p-mediated FBN1 inhibition. Mol Oncol. 2020;14(10):2660-77.

35. Nusse R, Clevers H. Wnt/beta-catenin signaling, disease, and emerging therapeutic modalities. Cell. 2017;169(6):985-99.

36. Vilchez V, Turcios L, Marti F, Gedaly R. Targeting Wnt/beta-catenin pathway in hepatocellular carcinoma treatment. World J Gastroenterol. 2016;22(2):823-32.

37. Fang $F$, VanCleave $A$, Helmuth $R$, Torres $H$, Rickel $K$, Wollenzien $H$, et al. Targeting the Wnt/beta-catenin pathway in human osteosarcoma cells. Oncotarget. 2018;9(95):36780-92.

38. Du Z, Li F, Wang L, Huang H, Xu S. Regulatory effects of microRNA184 on osteosarcoma via the Wnt/betacatenin signaling pathway. Mol Med Rep. 2018;18(2):1917-24.

39. Ma Y, Ren Y, Han EQ, Li H, Chen D, Jacobs JJ, et al. Inhibition of the Wntbeta-catenin and Notch signaling pathways sensitizes osteosarcoma cells to chemotherapy. Biochem Biophys Res Commun. 2013;431(2):274-9.

40. Wu BQ, Cao Y, Bi ZG. Suppression of adriamycin resistance in osteosarcoma by blocking Wnt/beta-catenin signal pathway. Eur Rev Med Pharmacol Sci. 2020;24(14):7548.

\section{Publisher's Note}

Springer Nature remains neutral with regard to jurisdictional claims in published maps and institutional affiliations. 